\title{
Locating landslides using multi-temporal satellite images
}

\author{
K.S. Cheng *, C. Wei, S.C. Chang \\ Department of Bioenvironmental Systems Engineering/Hydrotech Research Institute, National Taiwan University, \\ No. 1, Section 4, Taipei, Taiwan, ROC
}

Received 25 November 2002; received in revised form 16 April 2003; accepted 17 April 2003

\begin{abstract}
Landuse/landcover change detection using remotely sensed images has been widely investigated. Most applications of this type involve either image differencing or image classification using multi-temporal images. If multi-temporal images are to be used for quantitative analysis based on their radiometric information, as in the case of change detection or landuse classification, geometric rectification and radiometric correction must be performed priori to subsequent image analyses. In particular, geometric rectification has significant effect on the accuracy of landuse change detection in areas of rugged terrain. Remote sensing image rectification is commonly done by applying a polynomial trend mapping (PTM) model to image coordinates and map coordinates of groundcontrol-points. A major drawback of the PTM model is that it does not capture the random characteristics of terrain elevation. In this study an ordinary kriging approach is applied for image-to-image registration. The approach considers residuals of the PTM model as anisotropic random fields and employs the ordinary kriging method for spatial interpolation of the residual random fields. Band-ratioing technique was also employed for relative radiometric normalization. From the grey-level histograms of pre- and postevent band-ratio images, we determined the percentage of landuse changes in the study area. Image differencing was then performed using the pre- and post-event band-ratio image pair. Finally, a grey-level threshold of the band-ratio difference image is determined as the value whose exceeding probability equals the areal percentage of landuse change. DTM data of the study area were also used to further restrict landslide areas to steep slope areas.
\end{abstract}

(C) 2003 COSPAR. Published by Elsevier Ltd. All rights reserved.

Keywords: Change detection; Landslide; Remote sensing; Kriging

\section{Introduction}

Using remote sensing data and techniques for land surface change detection has gained increasing attentions in recent past. It is particularly so in Taiwan due to rapid development in hilly areas and frequent occurrences of natural disasters such as earthquakes, typhoons, and storm-induced debris flows. The ferocious Chi-Chi earthquake, that occurred on September 21, 1999 and measured at level 7.3 in Richter's scale, severely damaged several townships in Central Taiwan and drastically changed the landscape in extensive mountainous areas. After the earthquake, piled rocks and soils on the hilly areas become unstable and are vulnerable to intensive storms. As results, typhoon

\footnotetext{
${ }^{*}$ Corresponding author.

E-mail address: rslab@ntu.edu.tw (K.S. Cheng).
}

seasons of the following years have seen repeated occurrences of landslides and debris flows in the area claiming lives and properties of villagers. The Experimental Forest of the National Taiwan University (EFNTU) is among the most severely damaged areas. Limited by available man power, resorting to remote sensing techniques for landslide identification and restoration action planning is inevitable.

Remote sensing techniques of land surface change detection include post-classification comparison, temporal image differencing, temporal image ratioing, multifractal analysis, Bayesian probabilistic method (Lunetta and Elvidge, 1999), etc. Despite their differences in change detection algorithm, all change detection methods deal with multi-temporal images that are acquired in different dates and have different view angles, sun angles, atmospheric conditions, and spatial coverages. Therefore, a ground cell usually corresponds 
to different image coordinates in different images, and registration of all images to a common reference, be a map or a specific image, is a required pre-process for subsequent change detection. A large portion of misidentified land surface changes may occur due to inappropriate image registration. Unfortunately, many applications of change detection using remote sensing images do not pay particular attention to possible misidentification due to inappropriate image registration.

There are essentially two different categories of image rectification/registration approaches, the deterministic and statistical approaches. The deterministic approach, also known as the sensor distortion model, relies on data of the flight parameters and the terrain information, and is effective when types of distortion are well characterized (Richards, 1995). For many applications, such information and parameters may not be available making it difficult to adopt a deterministic approach of image registration. The statistical approach, by means of a set of ground-control-points (GCPs), establishes empirical relationship between image coordinates and their corresponding map/image coordinates using standard statistical procedures. The most widely used method in this category is the polynomial trend mapping (PTM) technique, also known as the polynomial distortion model, that employs polynomial regression equations to relate image coordinates and their corresponding map/image coordinates. Although commonly applied, the PTM technique often yields significant registration errors in mountainous or rugged terrain areas due to its inability to account for the local and random variation of terrain elevation. Cheng et al. (2000) proposed an anisotropic spatial modeling approach using ordinary kriging estimation for image rectification. The approach takes into account the spatial variation structure of terrain elevation and yields zero registration error at GCPs. It is believed that the kriging approach can be applied for multi-temporal image-to-image registration with high accuracy. Therefore, the objective of this study is to identify landslide sites with high accuracy using multitemporal satellite images registered by kriging approach.

\section{Study area and data}

The Experimental Forest of National Taiwan University locates in Central Taiwan and covers a total area of $333 \mathrm{~km}^{2}$. Terrain elevation in the area ranges from 220 to $3952 \mathrm{~m}$ above the mean sea level. A major northerly flowing stream confluents surface runoff to the Juo-Shuei River, one of the major rivers in Taiwan. The area is very close to the center of the Chi-Chi earthquake. SPOT multi-spectral images, acquired on October 1, 1999 and September 21, 2001, are used in this study for identification of landslide sites. A major typhoon (Toraji) passed through the study area on July
30, 2001 and induced landslides and debris flows. Orthorectified airphotos of scale 1:5000 and digital terrain model (DTM) data are also used for image rectification and calculation of land surface slope, respectively.

\section{Image registration and rectification}

Change detection using multi-temporal images requires all images be referenced to a common base, either be a map or an image, followed by a comparison of image radiances. If two images are utilized for change detection two approaches may be adopted. The first approach is to independently reference the two images to a common orthorectified map and then compare the radiance properties of both images. The second approach is to firstly rectify one of the two images to an orthorectified map and then perform an image-to-image registration between the two images.

The first approach will result in misidentified changes owing to rectification errors on the two images and the inconsistency of incorrectly rectified areas. Image-toimage registration is expected to achieve higher accuracy than image-to-map rectification since both images generally are of the same spatial resolution and have similar radiance pattern making it easier to identify terrain patterns and find more GCPs. This is particularly advantageous in this study since the earthquake has significantly changed terrain features in many mountainous areas, and selecting GCPs on an outdated map becomes very difficult. Therefore, the second approach is adopted in this study in light of achieving higher accuracy of change detection through image-to-image registration.

The most widely used method for georeferencing of remote sensing images is the PTM method. Let $(x, y)$ be the map or referenced-image coordinate of a pixel and $(X, Y)$ be its corresponding coordinate in the distorted image. Also let $(\widehat{X}, \widehat{Y})$ be estimate of the image coordinate $(X, Y)$ by a polynomial transformation. General form of the PTM algorithm is given in the following equation:

$\widehat{X}=\sum_{i=0}^{n} \sum_{j=0}^{i} a_{i j} x^{i-j} y^{j}$

$\widehat{Y}=\sum_{i=0}^{n} \sum_{j=0}^{i} b_{i j} x^{i-j} y^{j}$

Here $n$ is the order of the polynomial model and the coefficients $a_{i j}$ and $b_{i j}$ are estimated by least-squares regression analysis. The PTM is basically a statistical trend surface mapping model. Using a set of $m$ GCPs, $\left[\left(x_{k}, y_{k}\right) ;\left(X_{k}, Y_{k}\right)\right], k=1,2, \ldots, m$, it finds the best-fit surface for the GCP data set. Low-order polynomials are used to rectify images with less geometric distortions, like satellite images from a flat region. Geometric 
distortions like scale, translation, rotation, and skew effects can be modeled by an affine transformation $(n=1)$. Therefore, the affine transformation can be used for approximate correction of satellite sensor- and orbitrelated distortions. Application of the PTM technique to image rectification in areas with significant variation of terrain elevation requires using higher order polynomials and often leads to significant image distortions for regions outside the range of GCPs. Since image rectification in the PTM technique is based on the least squares regression, it does not account for the effect of local terrain variation on rectification accuracy, and inevitably yields rectification errors at all GCPs.

Cheng et al. (2000) proposed a geostatistical approach of image rectification that takes into account the effect of terrain variation on rectification accuracy and yields zero rectification error at all GCPs. The approach assumes that the spatial distribution of registration errors resulted from a low-order PTM model can be modeled as a random field which may be isotropic or anisotropic. Spatial variation structure of such a random field is then characterized by a variogram, and estimation of PTMbased registration errors at non-GCP locations are estimated by ordinary kriging. Finally, the estimated registration errors are added to the PTM estimates $(X, \widehat{Y})$ to yield the final rectified coordinates. Detailed theory of kriging estimation can be found in Journel and Huijbregts (1978) and Chilès and Delfiner (1999).

Procedures of ordinary kriging approach of imageto-image registration are as follows:

(1) Select a set of GCPs using multi-temporal images.

(2) Perform the first order PTM to establish trend relationship between image coordinates of the two images.

(3) Consider the registration errors in $\mathrm{E}-\mathrm{W}$ and $\mathrm{N}-\mathrm{S}$ directions as two residual random fields and separately perform the anisotropic variogram modeling for each random field.

(4) Apply the ordinary kriging estimation to the residual random fields using anisotropic variograms established in step (3).

(5) Final result of the image-to-image registration is obtained by adding ordinary kriging estimates of the residual fields to the trend field estimated by PTM.

The ordinary kriging approach of image registration/ rectification has the advantage of capturing local terrain variation and reducing the image distortion caused by terrain variation. Particularly, in the case of image-toimage registration for multi-temporal images of the same spatial resolution, many GCPs scattering around the study area can be used, and the fact of zero registration error at all GCPs guarantees better overall registration accuracy. Readers are referred to Cheng et al. (2000) for a complete description of the approach. Image-to-map rectification can be done in a similar way except that map coordinates are involved. Fig. 1 shows

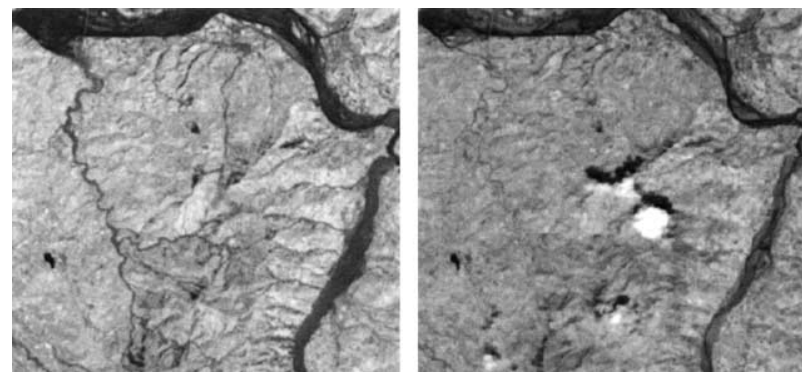

Fig. 1. IR band image pair after image-to-image registration.

result of the image-to-image registration by ordinary kriging approach for a portion of the study area.

\section{Change detection}

\subsection{Spectral ratioing}

Since multi-temporal images have different atmospheric conditions, spectral ratioing analysis is performed to effectively compensate for brightness variation caused by different atmospheric conditions. It can be seen in Fig. 1 that IR band brightness of the pre-typhoon image is lower than that of the post-typhoon image. After the IR/R band-ratioing, brightness values of the two band-ratio images are very close, as can be seen in Fig. 2 .

\subsection{Spectral histogram peaks matching}

Both spectral histograms of the pre- and post-typhoon band-ratio images have two correspondent peaks. Peaks in the histogram represent major landuse/landcover types and they should have stable grey levels in multitemporal band-ratio images. The histogram stretch technique was implemented to match correspondent histogram peaks. Fig. 3 shows spectral histograms of band-ratio images after histogram peaks matching.

\subsection{Estimating percentage of changed areas}

The histogram peaks matching yields pre- and postevent IR/R band-ratio images that have stable grey-level values for major landuse/landcover types. However, frequencies of specific grey levels are different in the two images. Frequency difference implies the number of pixels corresponding to landuse/landcover changes. Let $f_{1}(g)$ and $f_{2}(g)$, respectively, represent the frequency of grey level $g$ in pre- and post-event IR/R band-ratio images. The total number of pixels associated with landuse/ landcover changes, $N_{\mathrm{C}}$, and percentage of changed areas, $p$, are then estimated by the following equations:

$N_{\mathrm{C}}=\frac{1}{2} \sum_{g=1}^{256}\left|f_{1}(g)-f_{2}(g)\right|$, 

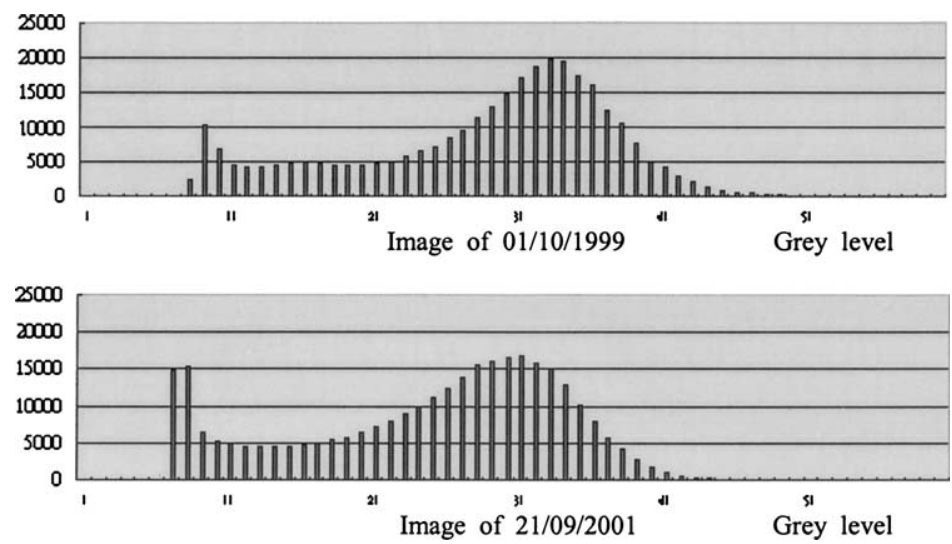

Fig. 2. Spectral histograms of IR/R band-ratio images.

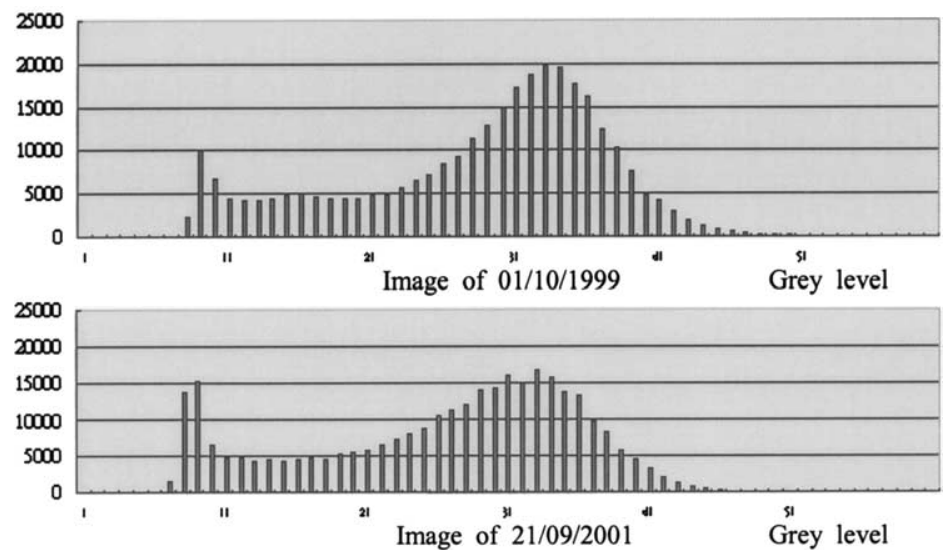

Fig. 3. Spectral histograms of IR/R band-ratio images after histogram peaks matching.

$p=100\left(N_{\mathrm{C}} / N\right)$

where $N$ is the total number of pixels in the image. Multiplying with $1 / 2$ in Eq. (2) is to avoid double counting of the number of changed pixels.

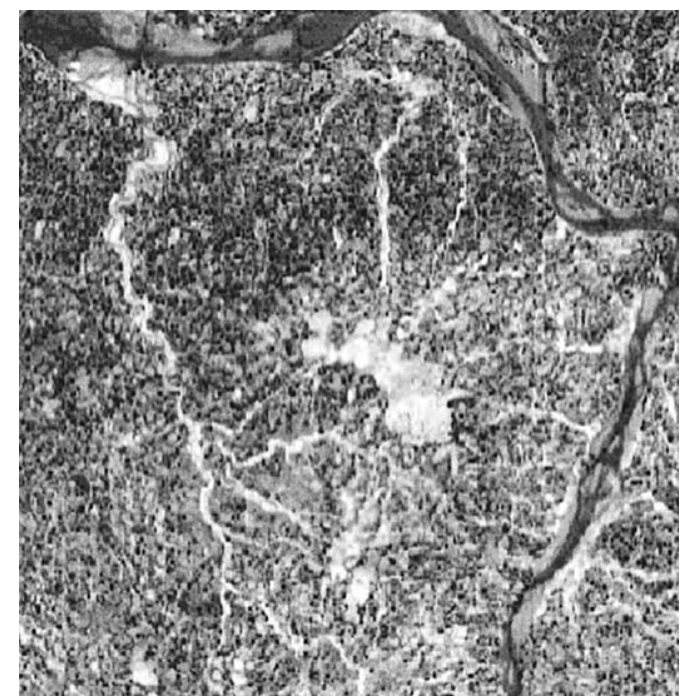

Fig. 4. IR/R band-ratio difference image.

\subsection{Determining a threshold for change detection}

Occurrences of landcover change correspond to significant difference in grey-level values of the pre- and post-event IR/R images. Therefore, multi-temporal image differencing was implemented using pre- and postevent IR/R band-ratio images to yield an IR/R bandratio difference image (see Fig. 4), hereafter referred to as the difference image. Grey level of pixels in the difference image, $g_{\text {diff }}$, is calculated by

$g_{\text {diff }}=\left|g_{\text {pre }}-g_{\text {post }}\right|$,

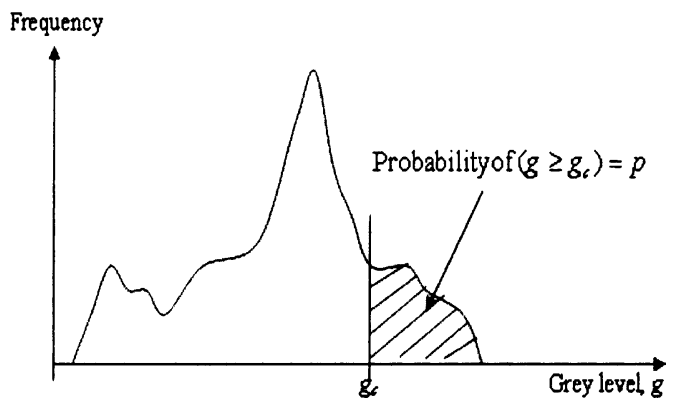

Fig. 5. Illustrative grey-level histogram of the difference image. 


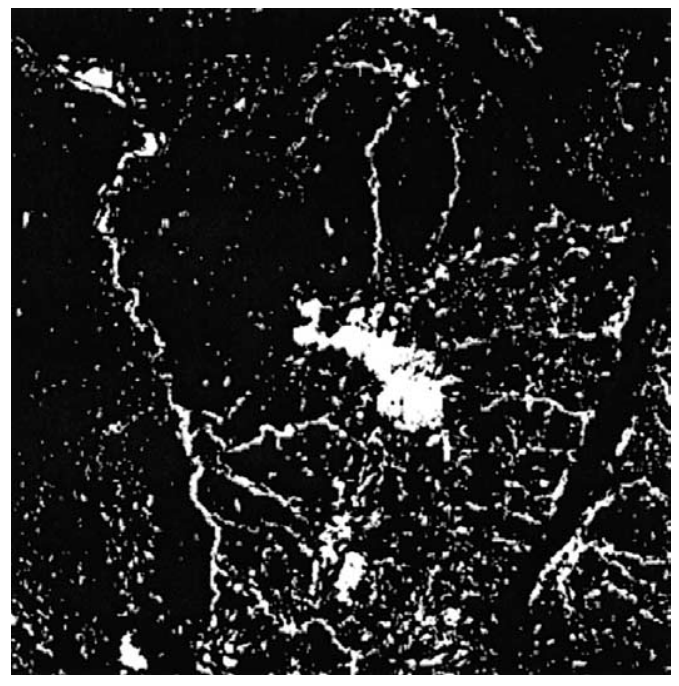

Fig. 6. Identified landcover-changed areas.

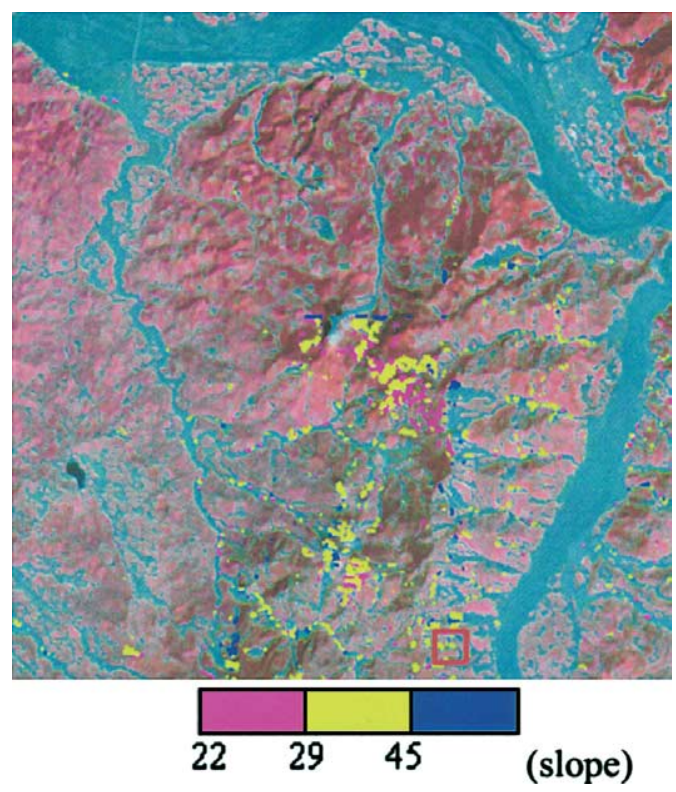

Fig. 7. Identified landslide sites. where $g_{\text {pre }}$ and $g_{\text {post }}$ are grey levels in the pre- and postevent band-ratio image, respectively. In the difference image, pixels associated with landcover changes have higher grey levels. A grey-level threshold, $g_{\mathrm{c}}$, for change detection using the difference image, is determined by locating the grey level whose exceeding probability equals the areal percentage of landuse changes, $p$, as depicted in Fig. 5. Changed areas identified by the greylevel threshold $g_{\mathrm{c}}$ are shown in Fig. 6 (white areas). Since not all change-detected areas are landslide sites, DTM data were used to generate a slope image of the study area and landslide identification is restricted to the area with slope steeper than $22^{\circ}$. Using $22^{\circ}$ as an empirical threshold for identifying landslides is based on previous studies of slopes of landslide sites in a Taiwan's watershed. Fig. 7 shows the identified landslide sites in the study area. Many identified landslide sites have been verified by field investigations and high resolution airphotos. For example, Fig. 8 shows the airphotos taken before and after the occurrence of Typhoon Toraji of an identified landslide site corresponding to the red blocked area in Fig. 7. It can be seen clearly that landslide occurred in a large area on the left half of Fig. 8(a) after Typhoon Toraji. Also, the large white cluster near the center in Fig. 6 represents a cloud covered area and therefore identified landslides in this region must be excluded. Although the threshold value is adopted to ensure high accuracy of identified landslides; however, it is also recognized that landslides may occur in lower slope areas.

\section{Conclusion}

In this study we demonstrate that the ordinary kriging approach of image-to-image registration yields high registration accuracy in mountainous and rugged terrain areas. The spectral ratioing technique yields similar brightness values for multi-temporal SPOT images and these multi-temporal band-ratio images can be used to estimate the percentage of landcover changes in the

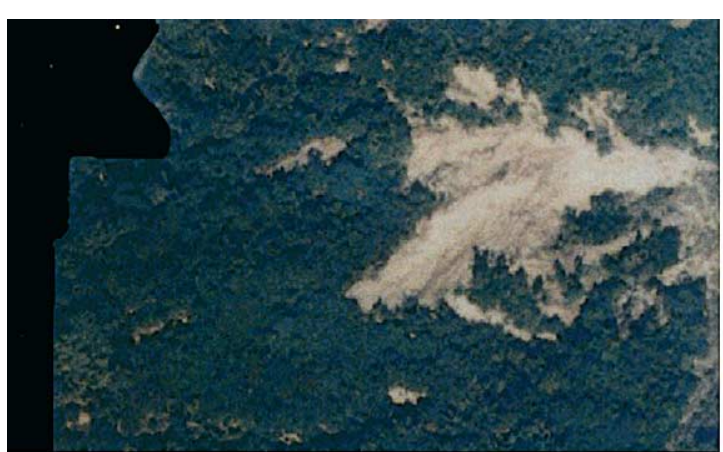

(a)

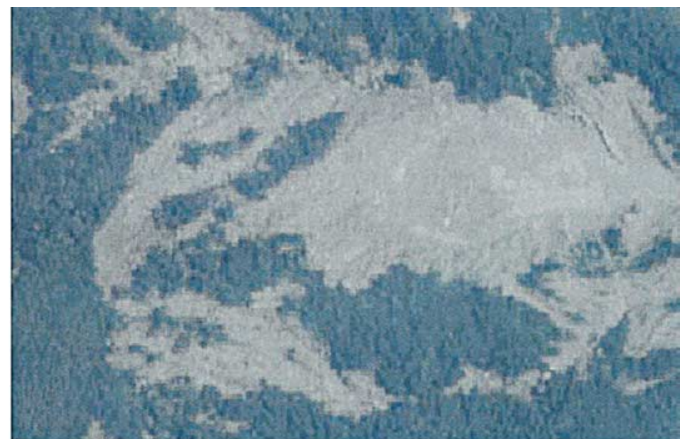

(b)

Fig. 8. Airphotos taken before and after Typhoon Toraji of an identified landslide site. 
study area. The multi-temporal image differencing technique yields a band-ratio difference image which can be used to determine the grey-level threshold for change detection. It is also demonstrated in this study that DTM data are very valuable in extracting landslide sites from the detected landcover-changed areas.

\section{Acknowledgements}

The authors are grateful for the financial support of a project by the Council of Agriculture of Republic of China. Special thanks are also given to staff members of the Experimental Forest of National Taiwan University, in particular, Professor Y.N. Wang. We also acknowl- edge the constructive comments and suggestions provided by reviewers.

\section{References}

Cheng, K.S., Yeh, H.C., Tsai, C.H. An anisotropic spatial modeling approach for remote sensing image rectification. Remote Sens. Environ. 73 (1), 46-54, 2000.

Chilès, J.P., Delfiner, P. Geostatistics - Modeling Spatial Uncertainty. Wiley, New York, pp. 449-451, 1999.

Journel, A.G., Huijbregts, C.J. Mining Geostatistics. Academic Press, London, 600 p, 1978.

Lunetta, R.S., Elvidge, C.D. Remote Sensing Change Detection. Taylor \& Francis Ltd., London, 318 p, 1999.

Richards, J.A. Remote Sensing Digital Image Analysis. Springer, Berlin, pp. 54-57, 1995. 